\title{
锌在林格氏液中的体外长期腐蚀降解行为
}

\author{
唐 帅，张文泰，钱军余，鲜鹏，莫小山，黄 楠，万国江 \\ (西南交通大学 材料科学与工程学院, 材料先进技术教育部重点实验室, 成都 610031)
}

摘 要: 锌基可降解生物材料与已被广泛研究的生物可降解材料(镁和铁)相比, 具有更合适的生物降解速率，因而 近年来受到了广泛的研究和关注。然而, 锌在模拟体液中的长期腐蚀降解行为尚不明确。本研究采用电化学腐蚀测 试、表面化学成分分析及降解模式演变观察相结合的方法，系统研究了锌在林格氏液中浸泡 $56 \mathrm{~d}$ 的腐蚀演化过程。 根据电化学结果显示，锌的腐蚀速率 $P_{\mathrm{i}}$ 在浸泡过程中基本保持稳定，约为 $0.06 \sim 0.10 \mathrm{~mm} / \mathrm{a}$; 失重法测定腐蚀速率为 $0.3 \mathrm{~mm} / \mathrm{a}$ 到 $0.5 \mathrm{~mm} / \mathrm{a}$ 。浸泡过程中生成的腐蚀产物主要为 $\mathrm{Zn}_{5}\left(\mathrm{CO}_{3}\right)_{2}(\mathrm{OH})_{6}$ 和 $\mathrm{CaCO}_{3}$, 为较致密的细条花棒状和块 状产物层, 且随着浸泡时间延长逐渐累积。去除腐蚀产物后发现, 样品表面出现较严重的局部腐蚀, 且腐蚀沟槽的 尺寸随浸泡时间的延长而增大, 浸泡 $42 \mathrm{~d}$ 腐蚀沟槽宽约为 $10 \mu \mathrm{m}$ 。本研究为锌基可降解生物材料后期表面改性及 潜在生物医学应用提供了数据积累和研究基础。

关 键 词: 可降解锌; 长期腐蚀行为; 电化学测试; 表面化学

中图分类号: TG178; R318 文献标识码: A

\section{Long-term in Vitro Corrosion Behavior of Zinc in Ringer's Solution}

\author{
TANG Shuai, ZHANG Wentai, QIAN Junyu, XIAN Peng,
} MO Xiaoshan, HUANG Nan, WAN Guojiang

(Key Laboratory of Advanced Technologies of Materials, Ministry of Education, School of Materials Science and Engineering, Southwest Jiaotong University, Chengdu 610031, China)

\begin{abstract}
In recent years, zinc-based biodegradable materials have gained significant attention due to their desirable biodegradation rate compared with other extensively explored biodegradable metals, such as magnesium and iron. However, the long-term corrosion behavior of zinc in simulated body fluid remain unclear. In this study, we performed a $56 \mathrm{~d}$ immersion test to reveal the long-term evolution of corrosion behavior of zinc in Ringer's solution using electrochemical methods and surface analysis. The results showed that the corrosion rate of $\mathrm{Zn}$ calculated from current density ranged from 0.06 to $0.1 \mathrm{~mm} / \mathrm{a}$ during the immersion. Its corrosion rate, determined by weight loss method, was from $0.3 \mathrm{~mm} / \mathrm{a}$ to $0.5 \mathrm{~mm} / \mathrm{a}$. The corrosion products were mainly composed of $\mathrm{Zn}_{5}\left(\mathrm{CO}_{3}\right)_{2}(\mathrm{OH})_{6}$ and $\mathrm{CaCO}_{3}$. These products were firm, rod- and block-like formed on Zn surface, and gradually accumulated with increase of immersion time. Its surface morphology after removing corrosion products exhibited increasing sizes of corrosion pits and grooves with increase of immersion time. And width of the corrosion pits and grooves was about $10 \mu \mathrm{m}$ after $42 \mathrm{~d}$ immersion. This study provides a guideline for the further surface modification and biomedical applications of
\end{abstract}

收稿日期: 2019-04-25; 收到修改稿日期：2019-06-07

基金项目：国家重点研发计划(2016YFC1102500); 国家自然科学基金(21473138)

National Key Research and Development Program of China (2016YFC1102500); National Natural Science Foundation of China (21473138)

作者简介：唐 帅(1992-), 男, 硕士研究生. E-mail: tangshuaicc@163.com TANG Shuai (1992-), male, Master candidate. E-mail: tangshuaicc@163.com

通讯作者: 万国江，教授. E-mail: guojiang.wan@home.swjtu.edu.cn WAN Guojiang, professor. E-mail: guojiang.wan@home.swjtu.edu.cn 
Zn-based materials in terms of biodegradation profile.

Key words: biodegradable zinc; long-term corrosion behavior; electrochemical test; surface chemistry

近年来，锌基金属与其它可降解金属相比，具 有更合适的生物降解速率的优势, 正成为最具潜力 的生物可降解植入材料 ${ }^{[1-2]}$ 。锌是人体内第二位必需 微量元素, 成人和贞儿的锌推荐日摄入量分别为 $8 \sim 11 \mathrm{mg} / \mathrm{d}$ 和 2 3 mg/d $\mathrm{d}^{[3]}$, 因此锌植入物在体内降 解过程中产生的锌离子安全浓度窗口大, 不易产 生系统毒性，可适应不同服役部位和生理环境。同 时, 锌参与了众多生理代谢过程, 具有一定的生理 功能 ${ }^{[4-6]}$, 包括信号传导、细胞调亡调节、抗动脉粥 样硬化和促成骨矿化以及骨细胞生长和愈合等。当 前针对锌基材料力学性能不足的合金化工作已取得 了诸多进展, 大量合金体系已可以满足临床力学性 能要求 ${ }^{[7-9]}$ 。锌 $\left(-0.762 \mathrm{~V}_{(\mathrm{SHE})}\right)$ 的标准电极电位介于镁 $\left(-2.34 \mathrm{~V}_{(\mathrm{SHE})}\right)$ 和铁 $\left(-0.44 \mathrm{~V}_{(\mathrm{SHE})}\right)$ 之间, 相对于镁在 体内的降解速率过快和铁的降解速率过慢, 锌在理 论上具有更适宜的生物降解速率。

一般来说, 生物可降解植入体需在人体内停留 3 6 个月甚至更长时间 ${ }^{[10]}$, 深入了解其在植入部位 服役过程中的降解行为十分重要, 而利用体外模拟 生理环境预测锌在体内的长期腐蚀行为是一种行之 有效的方法, 同时也对进一步的表面改性提供理论 基础。陈英奇等 ${ }^{[11]}$ 对比了镁、锌和铁在磷酸盐缓冲 液中的降解行为, 发现暂态测试中锌的开路电位和 降解速率位于镁和铁之间, 而浸泡过程中, 锌的降 解速率比镁和铁快, 这与理论不一致。而 Karin 等 ${ }^{[12]}$ 研究了纯锌在盐溶液、血浆和全血中 $3 \mathrm{~d}$ 的降解行 为, 发现血浆和全血中样品均匀腐蚀的表面上主要 是由无机盐和有机分子组成的腐蚀产物; 磷酸盐缓 冲液中样品为局部腐蚀, 表面主要由磷酸锌组成的 厚而多孔的腐蚀产物层; 而林格氏液中, 样品表面 浅坑界面上形成了碳酸锌凝胶状产物层, 更接近于 血浆和全血中的降解行为, 故对于锌的体外研究来 说, 林格氏液要优于磷酸盐缓冲液。近期越来越多 的研究开始关注纯锌在模拟体液中的腐蚀行为 ${ }^{[13-15]}$, 然而大多数只局限于短期 $(<14 \mathrm{~d})$ 腐蚀降解行为, 缺 乏长期腐蚀降解过程中电化学腐蚀、腐蚀产物演变、 腐蚀模式和腐蚀机制等方面的系统研究。

基于此, 本研究采用长期浸泡 (56 d)的方式, 研 究锌在模拟体液 (林格氏液) 中的腐蚀演化行为, 包 括电化学腐蚀演化规律、表面腐蚀产物分析和腐蚀 产物去除后的腐蚀模式演化。

\section{1 实验方法}

\section{1 样品制备}

将纯锌棒( $(99.99 \%$ )切成直径 $10 \mathrm{~mm}$, 厚度 $1.8 \mathrm{~mm}$ 的圆片，依次用 $600 \# 、 1000 \# 、 1500$ \#和 2000 \#的 SiC 砂纸研磨纯锌表面, 用酒精和去离子水超声清洗打 磨后的锌片(每次 $5 \mathrm{~min}$, 共 3 次), 最后用洗耳球吹 干置于真空干燥箱中备用。本研究中电解质溶液为 林格氏液 $\left(9 \mathrm{~g} / \mathrm{L} \mathrm{NaCl}, 0.43 \mathrm{~g} / \mathrm{L} \mathrm{KCl}, 0.2 \mathrm{~g} / \mathrm{L} \mathrm{NaHCO}_{3}\right.$, $0.24 \mathrm{~g} / \mathrm{L} \mathrm{CaCl}_{2}$ ), 所有试剂均为分析纯。林格氏液的 $\mathrm{pH}$ 为 $7.4 \pm 0.2$ 。

\section{2 电化学腐蚀表征}

采用 IM6 电化学工作站测量样品动电位极化曲 线(Potentiodynamic polarization curves, PDP)和电化 学阻抗谱(Electrochemical Impedance Spectroscopy, EIS)表征电化学腐蚀。电化学测试采用三电极系统 装置, 包括饱和甘录参比电极(Saturated Calomel Electrode, SCE), 铂对电极(Platinum sheet with dimensions of $1.5 \mathrm{~cm} \times 1.5 \mathrm{~cm}$ ) 和工作电极 $(\mathrm{Zn} \mathrm{sam}$ ples)。工作电极样品背面通过铜导线连接电化学工 作站, 并用硅橡胶密封, 暴露待测试表面面积为 $0.79 \mathrm{~cm}^{2}$ 。在 PDP 和 EIS 测试前, 样品在林格氏液 中浸泡至少 $30 \mathrm{~min}$, 以确保其表面处于稳定状态, 然后置于 $(37 \pm 0.5){ }^{\circ} \mathrm{C}$ 水浴锅中进行测试。对于动电 位极化测试，电位扫描区间为开路电位正负 $0.5 \mathrm{~V}$, 扫描速度为 $1 \mathrm{mV} / \mathrm{s}$ 。采用塔菲尔外推法对极化曲线 进行计算, 得到样品的腐蚀电位 $E_{\mathrm{corr}}$ 、腐蚀电流密 度 $i_{\text {corr }}$ 和阴极塔菲尔斜率 $\beta_{\mathrm{c}}$ 。根据标准 $\mathrm{ASTM}$

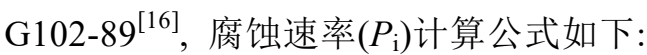

$$
P_{\mathrm{i}}=14.93 i_{\text {corr }}
$$

其中 $P_{\mathrm{i}} 、 i_{\text {corr }}$ 分别表示腐蚀速率 $(\mathrm{mm} / \mathrm{a})$ 和腐蚀电 流密度 $\left(\mathrm{mA} / \mathrm{cm}^{2}\right)$ 。电化学阻抗谱测试频率范围为 $100 \mathrm{kHz}$ 到 $0.01 \mathrm{~Hz}$, 测试所得数据用软件进行拟合 分析。每组样品重复 4 次电化学测试以符合统计学 要求。

\section{3 浸泡降解实验}

将打磨后的样品浸泡在 $(37 \pm 0.5){ }^{\circ} \mathrm{C}$ 的林格氏液 中，在浸泡 7、14、28、42 和 $56 \mathrm{~d}$ 时依次取出部分 样品, 研究其长期降解演化行为。每 4 个平行样品 设为一组, 浸泡在 $400 \mathrm{~mL}$ 的林格氏液中, 每 $3 \mathrm{~d}$ 更 换一次溶液。浸泡结束后取出样品, 用去离子水清 
洗三次并置于真空干燥箱中备用。用上述方法测试 样品的电化学腐蚀行为。采用体式显微镜 (Stemi 2000-C, ZEISS, Germany)和场发射扫描电子显微镜 (SEM, Quanta FEG 250, FEI, USA) 表征样品形貌。 通过 $X$ 射线衍射(XRD) 和 $X$ 射线光电子能谱(XPS) 分析表面腐蚀产物的结构和成分。XRD 测试采用 $\mathrm{CuK} \alpha$ 射线源, $2 \theta$ 记录范围为 $10^{\circ} \sim 90^{\circ}$, 步长 $0.033^{\circ}$ 。 XPS 测试采用 $\mathrm{Al} \mathrm{K \alpha}(1486.6 \mathrm{eV}) \mathrm{X}$ 射线源。根据标 准 ASTM G1-03, 将样品浸泡在 $200 \mathrm{mg} / \mathrm{mL} \mathrm{CrO}_{3}$ 溶液 中 $\left(80{ }^{\circ} \mathrm{C}\right) 1 \mathrm{~min}$ 去除表面腐蚀产物, 并通过 SEM 观 察去除腐蚀产物后的表面形貌。将去除腐蚀产物前 后的样品浸泡在液氮中 $0.5 \mathrm{~h}$ 后, 脆断制作截面, 采 用 SEM 和 EDS 线扫描分析截面的形貌和腐蚀产物 的元素含量。对样品浸泡前与去除腐蚀产物后进行 称重, 并根据标准 ASTM G31-72 计算腐蚀速率 ${ }^{[17]}$, 公式如下:

$$
P_{\mathrm{w}}=8.74 \times 10^{4} \frac{W}{A \cdot t \cdot \rho}
$$

其中, $P_{\mathrm{w}} 、 W 、 A 、 t 、 \rho$ 分别表示腐蚀速率 $(\mathrm{mm} / \mathrm{a})$ 、失 重 $(\mathrm{g})$ 、暴露面积 $\left(\mathrm{cm}^{2}\right)$ 、浸泡时间 $(\mathrm{h})$ 和密度 $\left(\mathrm{g} / \mathrm{cm}^{3}\right)$ 。

\section{2 结果与讨论}

\section{1 电化学腐蚀行为与分析}

锌在林格氏液中浸泡不同时间后的动电位极化 (PDP)曲线如图 1 所示。锌的自腐蚀电位在浸泡前

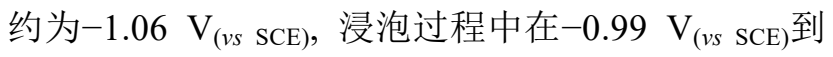
$-1.06 \mathrm{~V}_{(v s \mathrm{SCE})}$ 范围内波动。对于活性金属, 通过阴极 区极化曲线计算塔菲尔斜率更为可靠 ${ }^{[18]}$ 。计算结果显 示, 锌浸泡后的自腐蚀电流密度均比浸泡前的电流 密度小。在浸泡 $14 \mathrm{~d}$ 后, 腐蚀电流密度从 $42.7 \mu \mathrm{A} \cdot \mathrm{cm}^{-2}$ 逐步减小到 $6.2 \mu \mathrm{A} \cdot \mathrm{cm}^{-2}$, 并在浸泡 $28 \mathrm{~d}$ 后达到最低

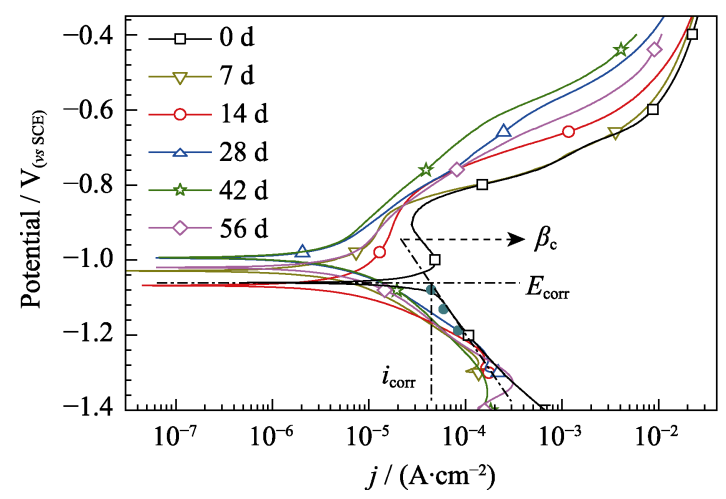

图 1 锌浸泡在 $(37 \pm 0.5)^{\circ} \mathrm{C}$ 林格氏液中 $7 、 14 、 28 、 42$ 和 $56 \mathrm{~d}$ 的 PDP 曲线

Fig. 1 Potentiodynamic polarization curves of $\mathrm{Zn}$ immersed in Ringer's solution at $(37 \pm 0.5){ }^{\circ} \mathrm{C}$ for $7,14,28,42$ and $56 \mathrm{~d}$
值 $4.9 \mu \mathrm{A} \cdot \mathrm{cm}^{-2}$, 在浸泡 42 和 $56 \mathrm{~d}$ 时保持稳定在 $5.7 \mu \mathrm{A} \cdot \mathrm{cm}^{-2}$ (图 2(a))。图 2(b)为根据自腐蚀电流密度 计算得出的腐蚀速率 $P_{\mathrm{i}}$, 相比于浸泡前, 锌浸泡后的 腐蚀速率明显降低，从 $0.64 \mathrm{~mm} / \mathrm{a}$ 下降到 $0.08 \mathrm{~mm} / \mathrm{y}$, 并在整个浸泡过程中保持稳定，约为 $0.06 \mathrm{~mm} / \mathrm{a}$ 到 $0.1 \mathrm{~mm} / \mathrm{a}$ 。

根据电化学实验结果可以看出, 浸泡过程中产 生的腐蚀产物层对基体具有一定的保护作用，显著 降低了锌的腐蚀速率, 并在整个浸泡过程中保持稳 定。通过极化曲线计算所得的锌在林格氏液中的降解 速率要高于临床上理想的降解速率标准 $0.02 \mathrm{~mm} / \mathrm{a}^{[1]}$, 而通过电化学方法估算样品的腐蚀速率是方法之一, 后面将通过失重法进一步分析腐蚀速率。

锌在林格氏液中浸泡不同时间后的电化学阻抗 谱如图 3 所示。从图 3(a)中可以看出, 锌在浸泡后 的阻抗环相较于浸泡前明显增大。随着浸泡时间的 延长, 样品阻抗环进一步增大。从阻抗 Bode 图和 Nyquist 图可以看出, 锌在浸泡前具有两个时间常 数并在低频区域出现扩散控制现象, 可能是由于锌 电极表面反应过快，电极表面附近反应物无法扩散， 因此反应由反应物从溶液本体扩散到电极表面的过 程控制 ${ }^{[19-20]}$ 。而当表面被腐蚀产物覆盖后，反应
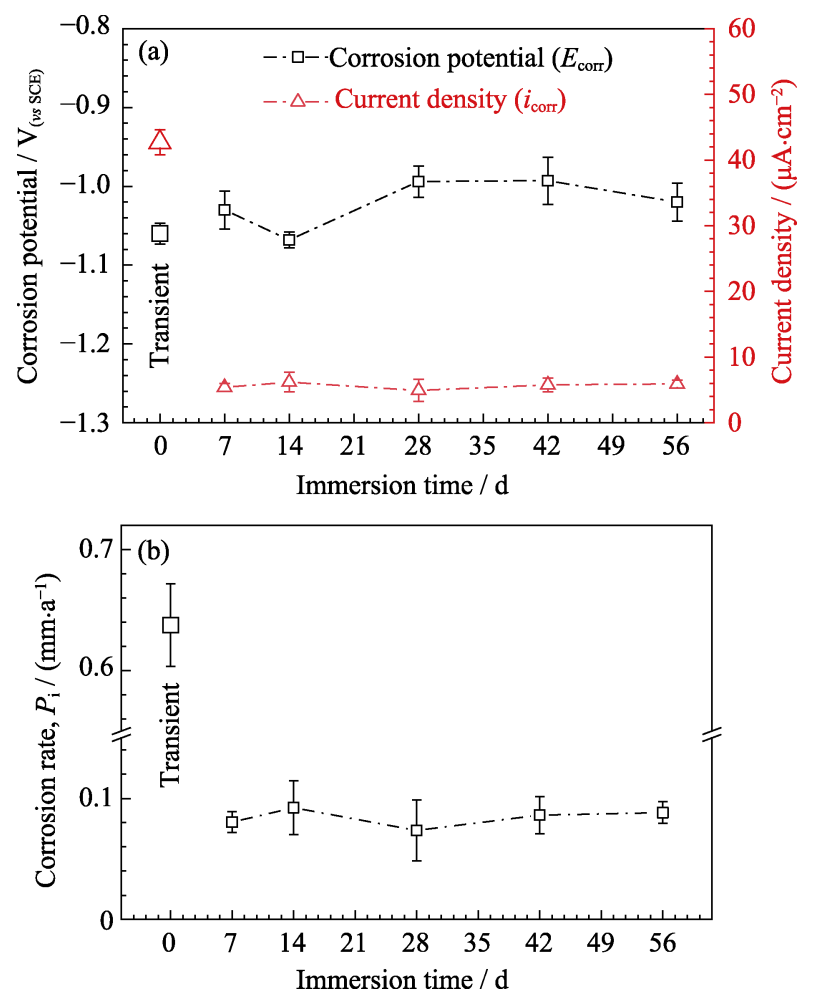

图 2 根据 PDP 曲线计算的自腐蚀电位 $E_{\text {corr }}$ 和自腐蚀电流 密度 $i_{\text {corr }}(\mathrm{a})$ 和根据自腐蚀电流密度计算的腐蚀速率 $P_{\mathrm{i}}(\mathrm{b})$

Fig. 2 Parameters of corrosion potential and corrosion current density obtained from PDP curves (a), and corrosion rate $P_{\mathrm{i}}$ obtained from current density (b) 

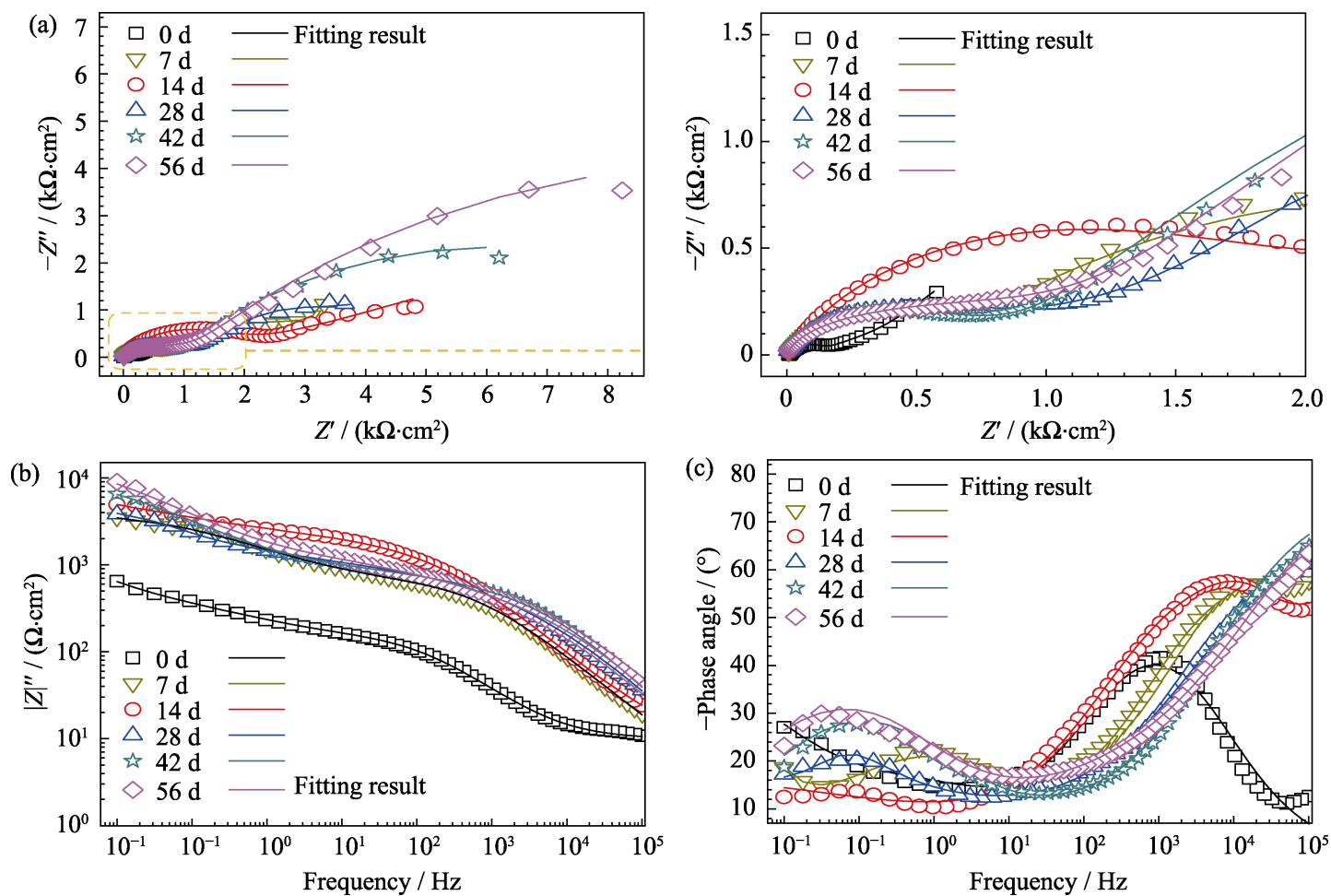

图 3 锌浸泡在 $(37 \pm 0.5){ }^{\circ} \mathrm{C}$ 下的林格氏液中 7、14、28、42 和 $56 \mathrm{~d}$ 后 EIS 测试结果

Fig. 3 Nyquist plots measured by Electrochemical Impedance Spectroscopy (EIS) (a), bode plots of $|Z| v s$. frequency (b) and bode plots of phase angle $v s$. frequency (c) of $\mathrm{Zn}$ immersed in Ringer's solution at $(37 \pm 0.5){ }^{\circ} \mathrm{C}$ for $7,14,28,42$ and $56 \mathrm{~d}$

速率下降，扩散控制现象消失，取而代之的是中频 区域时间常数的增大, 可能是由于腐蚀产物的分层 造成的。因此, 锌在浸泡前的阻抗曲线可以用电路 图 $R_{\mathrm{s}}\left(Q_{\mathrm{p} 1}\left(R_{\mathrm{p} 1}\left(Q_{\mathrm{dl}}\left(R_{\mathrm{ct}} Z_{\mathrm{w}}\right)\right)\right)\right)^{[15]}$ (图 4(a))进行拟合分析。 锌在浸泡过程中的阻抗曲线可以用电路图 $R_{\mathrm{s}}\left(Q_{\mathrm{p} 1}\right.$ $\left.\left(R_{\mathrm{p} 1}\left(Q_{\mathrm{p} 2}\left(R_{\mathrm{p} 2}\left(Q_{\mathrm{d} 1} R_{\mathrm{ct}}\right)\right)\right)\right)\right)$ (图 4(b))进行拟合分析。其中,

(a)
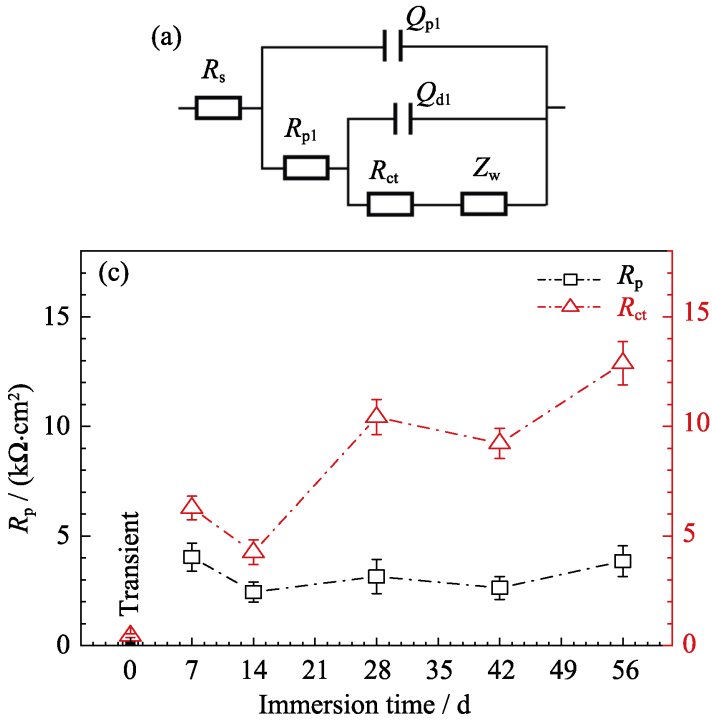

$R_{\mathrm{S}}$ 代表溶液电阻; $Q_{\mathrm{p} 1} 、 R_{\mathrm{p} 1}$ 和 $Q_{\mathrm{p} 2} 、 R_{\mathrm{p} 2}$ 分别代表外层 和内层腐蚀产物的电容和电阻; $Q_{\mathrm{dl}}$ 和 $R_{\mathrm{ct}}$ 分别代表 双电层的电容和电阻, 与界面电荷转移反应有关, $R_{\mathrm{ct}}$ 反映了抵抗腐蚀的程度; $Z_{\mathrm{w}}$ 表示扩散电阻。

根据电路图拟合出的动力学参数值如表 1 所 示。其中, 腐蚀产物电阻 $R_{\mathrm{p}}\left(R_{\mathrm{p} 1}+R_{\mathrm{p} 2}\right)$ 和双电层电阻

(b)
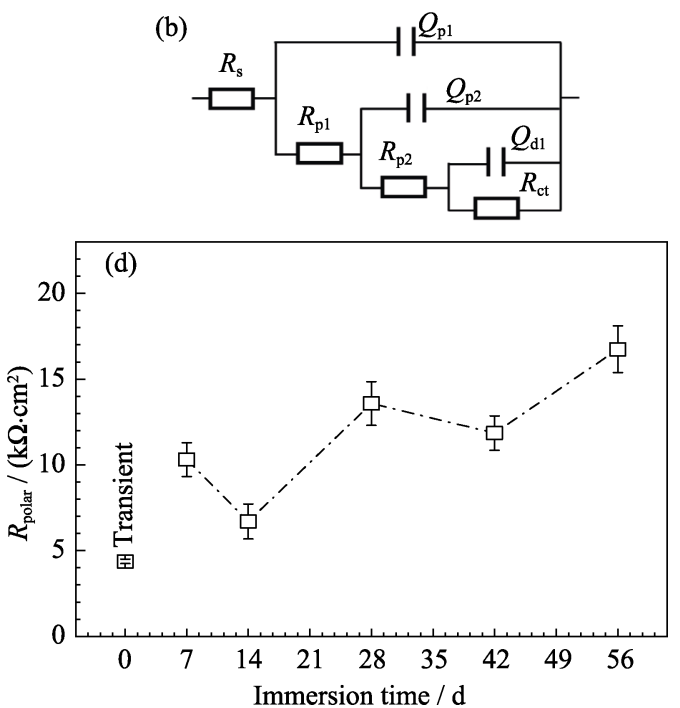

图 4 锌在浸泡前的 EIS 数据拟合电路图(a), 浸泡 7 56 d 后 EIS 数据拟合电路图(b), 界面电荷 转移电阻 $R_{\mathrm{ct}}$ 和腐蚀产物电阻 $R_{\mathrm{p}}$ 的拟合结果( $\left.\mathrm{c}\right)$, 极化电阻 $R_{\mathrm{polar}}$ 的拟合结果(d)

Fig. 4 EIS data fitted with Equivalent electrical circuit (EEC) for $0 \mathrm{~d}$ (a) and EEC for 7 to $56 \mathrm{~d}$ (b), interfacial charge transfer resistance $R_{\mathrm{ct}}$ and the corrosion products resistance $R_{\mathrm{p}}$ obtained from fitted results of the EIS spectra (c), and polarization resistance $R_{\text {polar }}$ calculated from EIS components as a function of time (d) 
表 1 锌在 $(37 \pm 0.5){ }^{\circ} \mathrm{C}$ 林格氏液中的 EIS 拟合结果

Table 1 The evolution of fitted results of electrochemical impedance spectroscopy of $\mathrm{Zn}$ in Ringer's solution at (37 \pm 0.5$){ }^{\circ} \mathrm{C}$

\begin{tabular}{|c|c|c|c|c|c|c|c|c|c|c|c|}
\hline Samples & $\begin{array}{c}R_{\mathrm{s}} / \\
\left(\Omega \cdot \mathrm{cm}^{2}\right)\end{array}$ & $\begin{array}{c}Q_{\mathrm{p} 1} /\left(\times 10^{-6},\right. \\
\left.\mathrm{s}^{n^{*}} \cdot \Omega^{-1} \cdot \mathrm{cm}^{-2}\right)\end{array}$ & $n_{1}$ & $\begin{array}{c}R_{\mathrm{p} 1} / \\
\left(\Omega \cdot \mathrm{cm}^{2}\right)\end{array}$ & $\begin{array}{c}Q_{n^{*} 2} /\left(\times 10^{-6}\right. \\
\left.\mathrm{s}^{n^{*}} \cdot \Omega^{-1} \cdot \mathrm{cm}^{-2}\right)\end{array}$ & $n_{2}$ & $\begin{array}{c}R_{\mathrm{p} 2} / \\
\left(\Omega \cdot \mathrm{cm}^{2}\right)\end{array}$ & $\begin{array}{c}Q_{\mathrm{dl}} /\left(\times 10^{-6},\right. \\
\left.\mathrm{s}^{n^{*}} \cdot \Omega^{-1} \cdot \mathrm{cm}^{-2}\right)\end{array}$ & $n_{3}$ & $\begin{array}{c}R_{\mathrm{ct}} /\left(\times 10^{3},\right. \\
\left.\Omega \cdot \mathrm{cm}^{2}\right)\end{array}$ & $\begin{array}{c}Z_{\mathrm{w}} /\left(\times 10^{-3}\right. \\
\left.\mathrm{s}^{1 / 2} \cdot \Omega^{-1} \cdot \mathrm{cm}^{-2}\right)\end{array}$ \\
\hline $0 \mathrm{~d}$ & 21.09 & 35.00 & 0.76 & 105 & - & - & - & 2376 & 0.80 & 0.45 & 3.81 \\
\hline $7 \mathrm{~d}$ & 22.48 & 5.05 & 0.71 & 576 & 315.0 & 0.49 & 3458 & 155 & 0.61 & 6.28 & - \\
\hline $14 \mathrm{~d}$ & 16.21 & 1.24 & 0.65 & 175 & 13.7 & 0.74 & 2266 & 798 & 0.56 & 4.26 & - \\
\hline $28 \mathrm{~d}$ & 21.28 & 16.80 & 0.72 & 260 & 344.0 & 0.22 & 2893 & 322 & 0.81 & 10.42 & - \\
\hline $42 \mathrm{~d}$ & 22.91 & 0.95 & 0.74 & 430 & 232.0 & 0.49 & 2200 & 243 & 0.82 & 9.22 & - \\
\hline $56 \mathrm{~d}$ & 23.56 & 2.71 & 0.70 & 511 & 121.0 & 0.50 & 3342 & 290 & 0.57 & 12.88 & - \\
\hline
\end{tabular}

*: s indicates the time unit "second", and $n$ indicates diffusion index

$R_{\mathrm{ct}}$ 随浸泡时间的趋势如图 4(c) 所示, 浸泡后腐蚀产 物电阻 $R_{\mathrm{p}}$ 和双电层电阻 $R_{\mathrm{ct}}$ 均远高于浸泡前的电阻。 随着浸泡时间的延长, 涂层总电阻未出现明显的变 化, 这一结果与极化计算所得结果相吻合, 说明腐 蚀产物层具有良好的稳定性。同时, 根据内外层腐 蚀产物层的拟合电阻可以看出, 锌表面生成的腐蚀 产物层是由致密的内层与相对疏松的外层构成。极 化电阻 $R_{\mathrm{polar}}$ 与双电层电阻 $R_{\mathrm{ct}}$ 可以反映样品整体的 抗腐蚀能力 ${ }^{[21]}$, 浸泡前极化电阻可由公式 $R_{\text {polar }}=$ $R_{\mathrm{ct}}+Z_{\mathrm{w}}+R_{\mathrm{p}}$ 算出, 浸泡后极化电阻可由公式 $R_{\mathrm{polar}}=$ $R_{\mathrm{ct}}+R_{\mathrm{p}}$ 算出, 随浸泡时间变化规律如图 4(d)所示。 $R_{\text {polar }}$ 与 $R_{\mathrm{ct}}$ 规律相似, 随着浸泡时间的延长, 样品的 腐蚀抵抗能力整体呈增加趋势, 这主要由于表面腐 蚀产物层的保护作用。

\section{2 腐蚀产物形貌及截面分析}

图 5 为锌在林格氏液中浸泡不同时间后的表面 形貌。从体式显微形貌照片可以看出, 随着浸泡时
间的延长, 表面腐蚀区域逐渐扩展到样品整个表面, 并伴随着白色腐蚀产物的积累与粗粮度的增加。整 个浸泡过程中未观察到锌表面出现严重的局部腐蚀 以及大量腐蚀产物堆积。从表面 SEM 照片可以看出, 锌在浸泡 $7 \mathrm{~d}$ 后的表面局部腐蚀产物主要呈细条花 棒状和块状。随着浸泡时间的延长, 表面腐蚀产物 逐步变成圆球小颗粒状和层片状，最后逐渐覆盖住 样品表面。

为了进一步观察腐蚀产物及被腐蚀产物覆盖的 基体的降解行为，对浸泡 $56 \mathrm{~d}$ 后的样品进行了截面 分析, 如图 6 所示。从图 6(a)的截面扫描电镜照片 可知，腐蚀产物层厚度约为 $60 \mu \mathrm{m}$, 并由表面到基 体逐渐变致密。图 6(b)为截面的 EDS 线扫数据图, 根据测试结果，可以明显地看出腐蚀产物层与基体 的分界点, 腐蚀产物层中主要包括 $\mathrm{Ca} 、 \mathrm{O} 、 \mathrm{C} 、 \mathrm{Zn}$ 四种元素, 可以看出元素随厚度变化的趋势, 但更 精确的表面成分需要结合后续的 XPS 结果来分析。
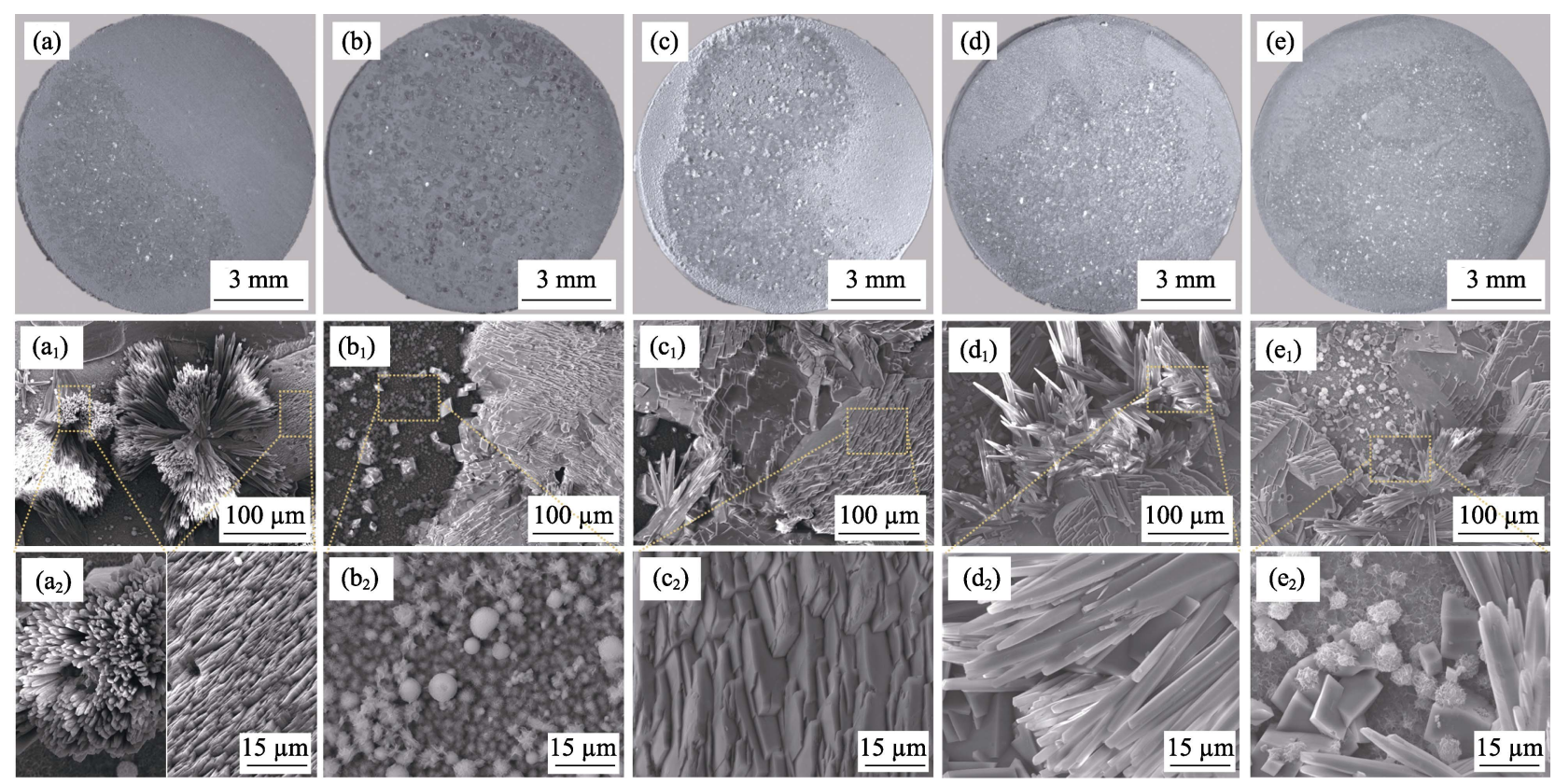

图 5 锌浸泡在 $(37 \pm 0.5){ }^{\circ} \mathrm{C}$ 的林格氏液中 7(a)、14(b)、28(c)、42(d)和 56(e) d 时的表面形貌

Fig. 5 Surface morphology of $\mathrm{Zn}$ immersed in Ringer's solution at $(37 \pm 0.5)^{\circ} \mathrm{C}$ for 7 (a), 14 (b), 28 (c), 42 (d), and 56 (e) d 

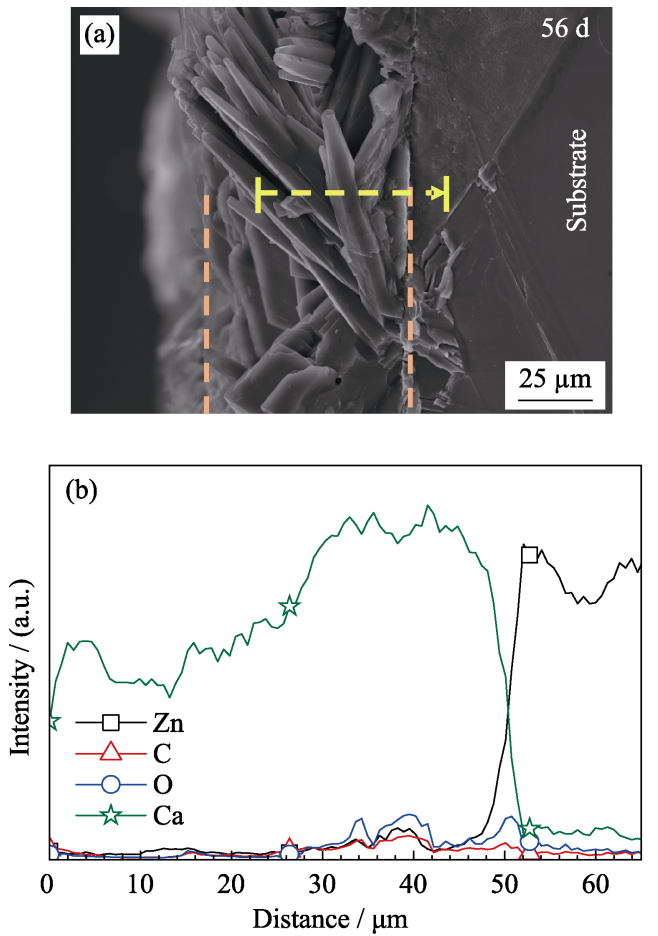

图 6 锌浸泡在 $(37 \pm 0.5){ }^{\circ} \mathrm{C}$ 林格氏液中 $56 \mathrm{~d}$ 的截面扫描电镜 照片(a)和截面 EDS 线扫图(b)

Fig. 6 Cross-sectional images (a) and EDS line profile (b) of $\mathrm{Zn}$ immersed in Ringer's solution at $(37 \pm 0.5){ }^{\circ} \mathrm{C}$ for $56 \mathrm{~d}$

从截面观察基体与腐蚀产物的界面层可知, 基体的 腐蚀为均匀腐蚀, 未观察到严重局部腐蚀坑。

\section{3 腐蚀产物结构及成分分析}

图 7 为浸泡不同时间后样品表面腐蚀产物的 $\mathrm{XRD}$ 图谱。从图中可以看出腐蚀产物主要为 $\mathrm{CaCO}_{3}$ 和 $\mathrm{Zn}_{5}\left(\mathrm{CO}_{3}\right)_{2}(\mathrm{OH})_{6}$, 并且两种腐蚀产物的量随着浸 泡时间的延长而增多。这一结果与表面形貌观察相 吻合, 浸泡过程中材料表面首先形成致密的细条状 $\mathrm{CaCO}_{3}$ 腐蚀产物内层, 对基体起到良好的保护作 用。随着浸泡时间的延长, 圆球状 $\mathrm{Zn}_{5}\left(\mathrm{CO}_{3}\right)_{2}(\mathrm{OH})_{6}$ 腐蚀产物逐渐积累, 并与 $\mathrm{CaCO}_{3}$ 混合形成疏松的外

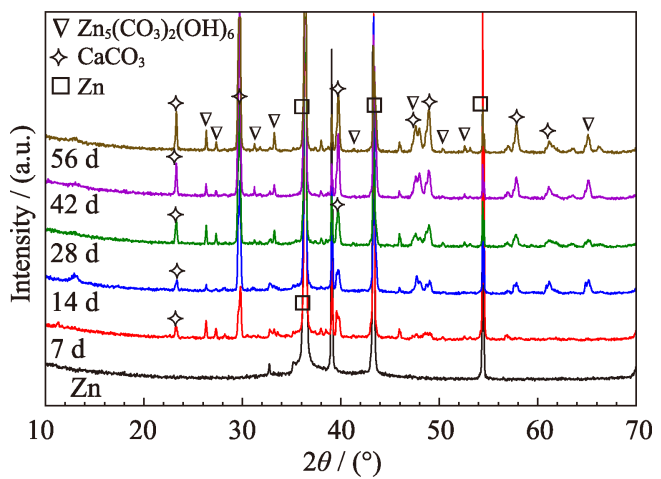

图 7 锌浸泡在 $(37 \pm 0.5){ }^{\circ} \mathrm{C}$ 林格氏液中 $7 、 14 、 28 、 42$ 和 $56 \mathrm{~d}$ 的 XRD 图谱

Fig. 7 XRD spectra of $\mathrm{Zn}$ immersed in Ringer's solution at $(37 \pm 0.5){ }^{\circ} \mathrm{C}$ for $7,14,28,42$, and $56 \mathrm{~d}$

\section{层腐蚀产物。}

为进一步表征腐蚀产物化学键的结合状态, 对 浸泡 $56 \mathrm{~d}$ 样品和纯锌进行了 X 射线光电子能谱检测, 如图 8 所示。从图 8(a)全谱中观察到浸泡 $56 \mathrm{~d}$ 样品 与纯锌比, 新增了 $\mathrm{Ca} 2 \mathrm{p}$ 的特征峰, $\mathrm{Zn} 2 \mathrm{p}$ 峰强度明显 降低, $\mathrm{C} 1 \mathrm{~s}$ 峰强度明显增强, 表明腐蚀产物中 $\mathrm{Ca} 、 \mathrm{C}$ 含量增加。图 8(b)进一步对 $\mathrm{O} 1 \mathrm{~s}$ 高分辨进行了分峰 处理, 发现浸泡 $56 \mathrm{~d}$ 后在 $529.1 \mathrm{eV}$ 处出现 $\mathrm{CaCO}_{3}$ 的新峰。图 8(c)对 $\mathrm{Ca} 2 \mathrm{p}$ 高分辨进行了分峰处理, $56 \mathrm{~d}$ 样品出现了三个特征峰, 分别是 $350.6 \mathrm{eV}$ 出现了 $\mathrm{CaCO}_{3} 、 \mathrm{Ca} 2 \mathrm{p}_{1 / 2}$ 的峰; $347.2 \mathrm{eV}$ 出现了 $\mathrm{CaCO}_{3}$ 、 $\mathrm{Ca} 2 \mathrm{p}_{3 / 2}$ 的峰; $344.6 \mathrm{eV}$ 出现了 $\mathrm{CaO}$ 的峰。故 XPS 检 测结果进一步证明，腐蚀产物中含有碳酸钙盐等化 合物。
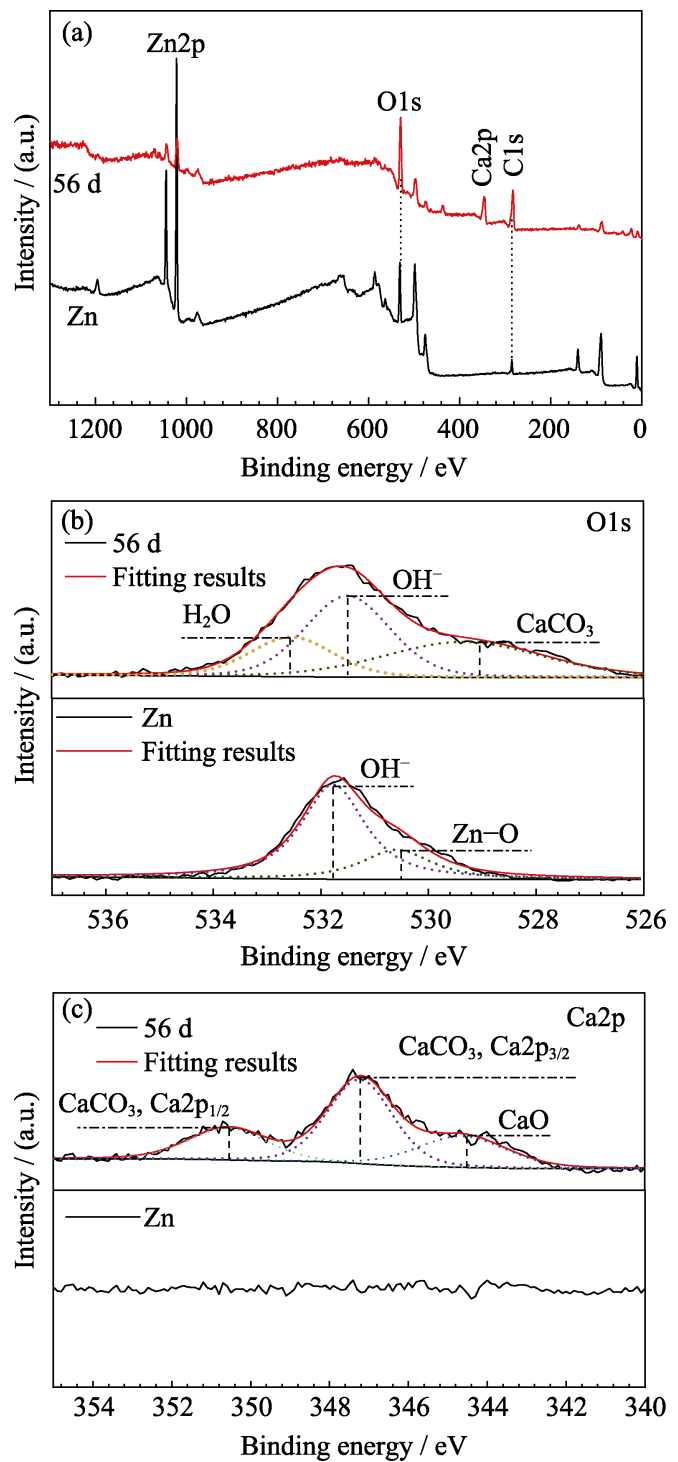

图 8 锌浸泡在林格氏液 $56 \mathrm{~d}$ 后和浸泡前的 XPS 图谱

Fig. 8 XPS spectra (a) and high-resolution O 1s (b) and (c) $\mathrm{Ca} 2 \mathrm{p}$ spectra of bare $\mathrm{Zn}$ and $\mathrm{Zn}$ after being immersed in Ringer's solution for $56 \mathrm{~d}$ 


\section{4 去除腐蚀产物分析}

图 9 为锌在林格氏液中浸泡不同时间后并去除 样品表面腐蚀产物后的 SEM 照片。从图中可以看出, 样品在浸泡 7 (图 9(a, a $\left.a_{1}\right)$ )和 $14 \mathrm{~d}\left(\right.$ (图 9(b, $\left.\left.\mathrm{b}_{1}\right)\right)$ 后, 表面 腐蚀较均匀, 腐蚀区域为河流状形貌，局部有微小 的腐蚀坑。随着浸泡时间的延长, 表面腐蚀坑逐渐增 大, 并在 $42 \mathrm{~d}$ (图 $9\left(\mathrm{~d}_{1} \mathrm{~d}_{1}\right)$ )时出现大约 $10 \mu \mathrm{m}$ 宽的裂纹。 到 $56 \mathrm{~d}$ (图 9(e, $\left.\mathrm{e}_{1}\right)$ ) 时, 裂纹进一步变长加深。这可 能由于浸泡前期先在含有杂质 $(\mathrm{Fe} 、 \mathrm{Ni}$ 等)处发生的 腐蚀, 随着浸泡时间的延长, 腐蚀产物逐渐覆盖腐 蚀坑, 到浸泡后期, 基体材料在少量穿透腐蚀产物
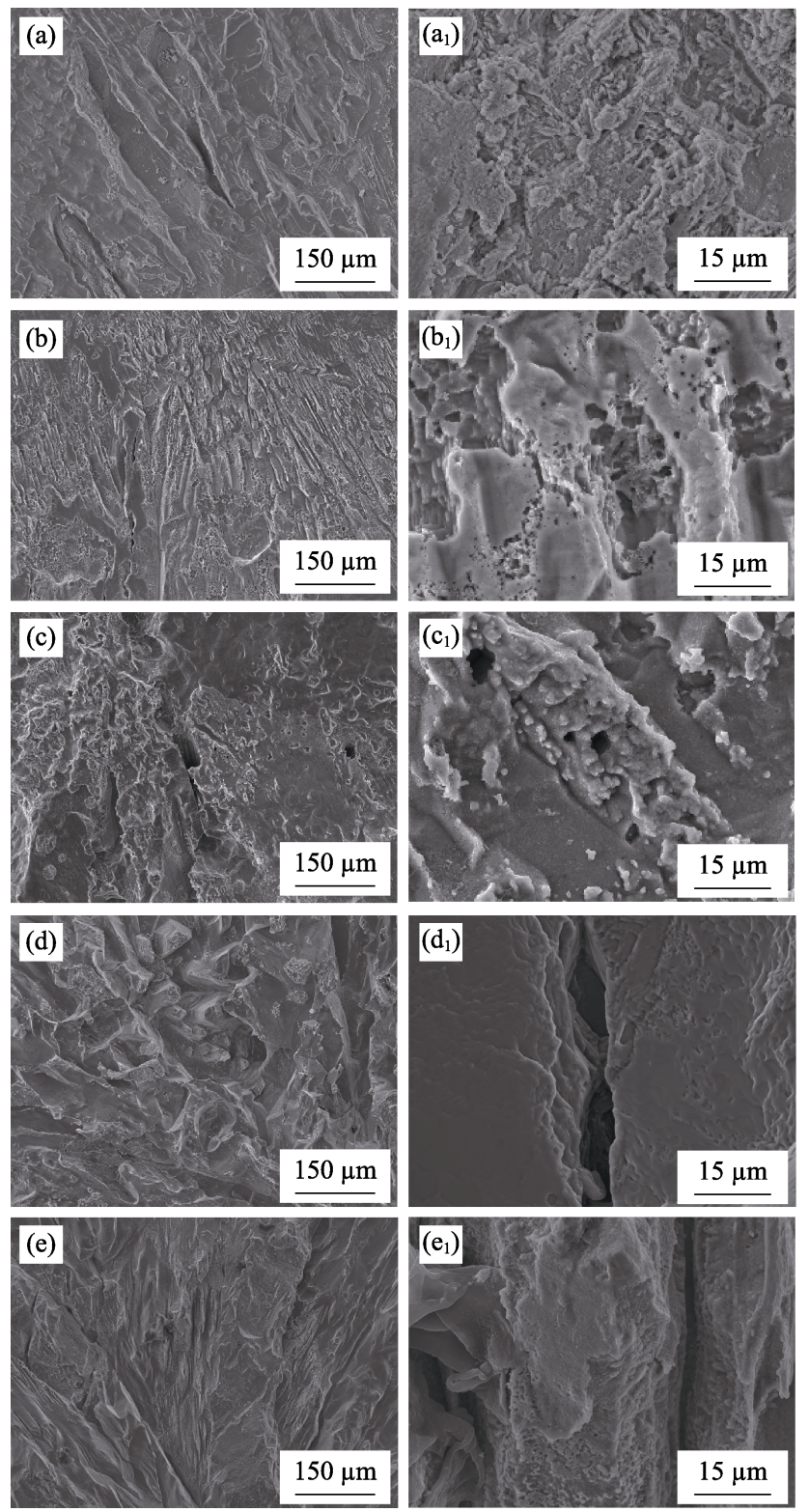

图 9 锌浸泡在 $(37 \pm 0.5){ }^{\circ} \mathrm{C}$ 林格氏液中 7(a)、14(b)、28(c)、 42(d)和 56(e)d 后去除腐蚀产物的表面形貌

Fig. 9 Surface morphologies of $\mathrm{Zn}$ immersed in Ringer's solution at $(37 \pm 0.5){ }^{\circ} \mathrm{C}$ for 7 (a), 14 (b), 28, (c) 42 (d), and 56 (e) d after removal of corrosion products
层的电解液作用下发生电偶腐蚀促进了腐蚀坑的纵 向发展, 形成了小孔腐蚀特征 ${ }^{[22-23]}$ 。图 10 为浸泡 $56 \mathrm{~d}$ 样品去掉腐蚀产物后的截面 SEM 照片, 可以看出 样品为局部腐蚀, 局部有腐蚀沟槽, 在沟槽里面有 微小的腐蚀坑。对比去除腐蚀产物前后的样品, 去 除腐蚀产物前表面表现为大体均匀腐蚀, 去除腐蚀 产物后基体表现为局部腐蚀, 说明去除腐蚀产物才 能更准确地分析基体的腐蚀模式。

\section{5 失重法腐蚀速率分析}

图 11 为失重法测量浸泡后的腐蚀速率 $P_{\mathrm{w}}$ 。锌 浸泡后的腐蚀速率大约为 $0.3 \mathrm{~mm} / \mathrm{a}$ 到 $0.5 \mathrm{~mm} / \mathrm{a}$ 之 间, 远远高于电化学计算所得腐蚀速率, 也显著高 于锌在其它溶液中的腐蚀速率 ${ }^{[11]}$ 。相比于根据极化 曲线计算出的腐蚀速率 $P_{\mathrm{i}}$, 在腐蚀速率大小上有差 别, 但随着浸泡时间的变化规律是一致的。两种方 法都比较准确地计算了锌在林格氏液中的腐蚀速率, 但电化学方法是测试样品浸泡后暂态的腐蚀速率, 而失重法是测试的整个浸泡过程的腐蚀速率, 故腐 蚀速率计算值不同。

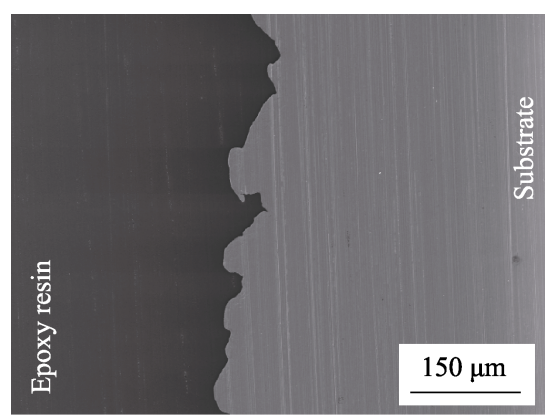

图 10 锌浸泡在 $(37 \pm 0.5){ }^{\circ} \mathrm{C}$ 林格氏液中 $56 \mathrm{~d}$ 去除腐蚀产物 后的截面扫描电镜照片

Fig. 10 Cross-sectional SEM images of $\mathrm{Zn}$ immersed in Ringer's solution at $(37 \pm 0.5){ }^{\circ} \mathrm{C}$ for $56 \mathrm{~d}$ after removal of corrosion products

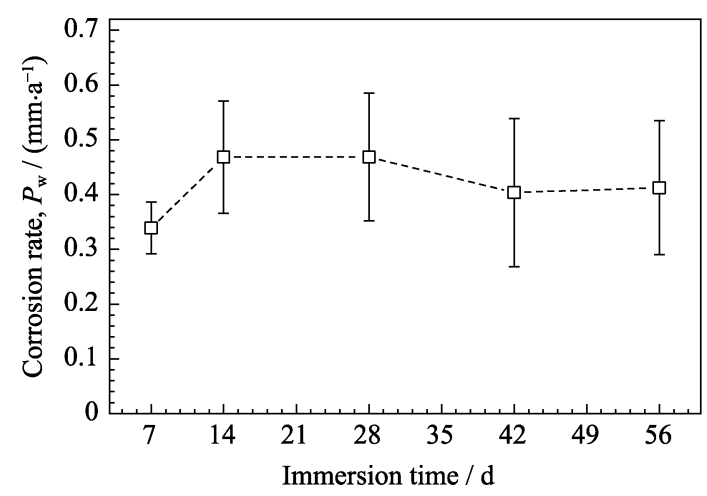

图 11 失重法测量锌浸泡在 $(37 \pm 0.5){ }^{\circ} \mathrm{C}$ 林格氏液中 $7 、 14$ 、 28、42 和 $56 \mathrm{~d}$ 的腐蚀速率

Fig. 11 Corrosion rate $P_{\mathrm{w}}$ calculated from weight loss of $\mathrm{Zn}$ after immersion in Ringer's solution at $(37 \pm 0.5){ }^{\circ} \mathrm{C}$ for 7,14 , 28,42 and $56 \mathrm{~d}$ 


\section{3 结论}

本研究采用电化学测试、表面形貌及成分分析 和去除腐蚀产物等方法, 对生物可降解锌金属在林 格氏液中的体外腐蚀降解演化行为进行了 $56 \mathrm{~d}$ 的长 期评价。结果表明，锌在林格氏液长期浸泡过程中 生成的腐蚀产物层由初期生成的相对致密的条块状 $\mathrm{CaCO}_{3}$ 内层及后期生成的较为疏松的 $\mathrm{CaCO}_{3}$ 和 $\mathrm{Zn}_{5}\left(\mathrm{CO}_{3}\right)_{2}(\mathrm{OH})_{6}$ 混合外层构成, 具有良好的保护作 用和稳定性。去除腐蚀产物后发现, 锌在林格氏液 中主要为局部腐蚀, 局部有腐蚀沟槽, 在沟槽里面 有微小的腐蚀坑，且随着浸泡时间的延长，腐蚀沟 槽增大。锌在林格氏液浸泡中的腐蚀速率通过失重 法计算为 $0.3 \sim 0.5 \mathrm{~mm} / \mathrm{a}$, 通过电化学方法测试为 0.06 到 $0.10 \mathrm{~mm} / \mathrm{a}$, 低于浸泡前的腐蚀速率, 说明浸 泡过程中腐蚀产物层对基体有一定的保护作用。

\section{参考文献:}

[1] BOWEN PATRICK K, DRELICH JAROSLAW, GOLDMAN JEREMY. Zinc exhibits ideal physiological corrosion behavior for bioabsorbable stents. Advanced Materials, 2013, 25(18): 2577-2582.

[2] BOWEN PATRICK K, SHEARIER EMILY R, ZHAO SHAN, et al. Biodegradable metals for cardiovascular stents: from clinical concerns to recent Zn-Alloys. Advanced Healthcare Materials, 2016, 5(10): 1121-1140.

[3] MOSTAED EHSAN, SIKORA-JASINSKA MALGORZATA, DRELICH JAROSLAW W, et al. Zinc-based alloys for degradable vascular stent applications. Acta Biomaterialia, 2018, 71: 1-23.

[4] BIN BUM-HO, BHIN JINHYUK, TAKAISHI MIKIRO, et al. Requirement of zinc transporter ZIP10 for epidermal development: implication of the ZIP10-p63 axis in epithelial homeostasis. Proceedings of the National Academy of Sciences, 2017, 114(46): 12243-12248.

[5] ZHU DONG-HUI, SU YING-CHAO, YOUNG MARCUS L, et al. Biological responses and mechanisms of human bone marrow mesenchymal stem cells to $\mathrm{Zn}$ and Mg biomaterials. ACS Applied materials \& Interfaces, 2017, 9(33): 27453-27461.

[6] HAASE HAJO, RINK LOTHAR. Multiple impacts of zinc on immune function. Metallomics, 2014, 6(7): 1175-1180.

[7] LIN SONG, WANG QI-LONG, YAN XIN-HAO, et al. Mechanical properties, degradation behaviors and biocompatibility evaluation of a biodegradable $\mathrm{Zn}-\mathrm{Mg}-\mathrm{Cu}$ alloy for cardiovascular implants. Materials Letters, 2019, 234: 294-297.

[8] KAFRI ALON, OVADIA SHIRA, GOLDMAN JEREMY, et al. The suitability of $\mathrm{Zn}-1.3 \% \mathrm{Fe}$ alloy as a biodegradable implant material. Metals, 2018, 8(3): 153.

[9] SHI ZHANG-ZHI, YU JING, LIU XUE-FENG, et al. Effects of Ag, $\mathrm{Cu}$ or $\mathrm{Ca}$ addition on microstructure and comprehensive properties of biodegradable $\mathrm{Zn}-0.8 \mathrm{Mn}$ alloy. Materials Science and Engineering: C, 2019, 99: 969-978.

[10] ZHENG YU-FENG, WU YUAN-HAO. Revolutionizing metallic biomaterials. Acta Metallurgica Sinica, 2017, 53(3): 257-297.

[11] CHEN YING-QI, ZHANG WEN-TAI, MAITZ MANFRED F, et al. Comparative corrosion behavior of $\mathrm{Zn}$ with $\mathrm{Fe}$ and $\mathrm{Mg}$ in the course of immersion degradation in phosphate buffered saline. Corrosion Science, 2016, 111: 541-555.

[12] TÖRNE KARIN, LARSSON MARIANN, NORLIN ANNA, et al. Degradation of zinc in saline solutions, plasma, and whole blood. Journal of Biomedical Materials Research Part B: Applied Biomaterials, 2016, 104(6): 1141-1151.

[13] ZHAO LI-CHEN, ZHANG ZHE, SONG YU-TING, et al. Mechanical properties and in vitro biodegradation of newly developed porous $\mathrm{Zn}$ scaffolds for biomedical applications. Materials \& Design, 2016, 108: 136-144.

[14] LIU LI-JUN, MENG YAO, DONG CHAO-FANG, et al. Initial formation of corrosion products on pure zinc in simulated body fluid. Journal of Materials Science \& Technology, 2018, 34(12): 2271-2282.

[15] LIU XIAO, YANG HONG-TAO, LIU YANG, et al. Comparative studies on degradation behavior of pure zinc in various simulated body fluids. JOM, 2019, 71(4): 1414-1425.

[16] STANDARD ASTM. G102-89, Standard Practice for Calculation of Corrosion Rates and Related Information from Electrochemical Measurements. Annual Book of ASTM Standards, ASTM International, West Conshohocken, PA, 2015.

[17] STANDARD ASTM. G31-72. Standard practice for laboratory immersion corrosion testing of metals. Annual Book of ASTM Standards, ASTM International, West Conshohocken, PA, 2004.

[18] SHI ZHI-MING, LIU MING, ATRENS ANDREJ. Measurement of the corrosion rate of magnesium alloys using Tafel extrapolation. Corrosion Science, 2010, 52(2): 579-588.

[19] HUANG JUN. Diffusion impedance of electroactive materials, electrolytic solutions and porous electrodes: Warburg impedance and beyond. Electrochimica Acta, 2018, 281: 170-188.

[20] WU J, ZHANG SD, SUN WH, et al. Influence of oxidation related structural defects on localized corrosion in HVAF-sprayed Fe-based metallic coatings. Surface and Coatings Technology, 2018, 335: 205-218.

[21] SHI ZHI-MING, CAO FU-YONG, SONG GUANG-LING, et al. Low apparent valence of $\mathrm{Mg}$ during corrosion. Corrosion Science, 2014, 88: 434-443.

[22] SIMõES AM, BASTOS AC, FERREIRA MG, et al. Use of SVET and SECM to study the galvanic corrosion of an iron-zinc cell. Corrosion Science, 2007, 49(2): 726-739.

[23] BLANDA GIUSEPPE, BRUCATO VALERIO, PAVIA FRANCESCO CARFì, et al. Galvanic deposition and characterization of brushite/ hydroxyapatite coatings on 316L stainless steel. Materials Science and Engineering: C, 2016, 64: 93-101. 